\title{
Spatial frameworks in imagined navigation
}

\author{
MARIOS N. AVRAAMIDES and SOFRONIS G. SOFRONIOU \\ University of Cyprus, Nicosia, Cyprus
}

\begin{abstract}
The spatial framework model proposes that people use the extensions of their body axes as a reference frame for encoding spatial layouts in memory, and that the physical and functional properties of our bodies and the world determine the accessibility of egocentric locations from memory representations. The present experiment provides evidence that spatial framework results can be obtained even with perceptual scenes that contain no objects to be held in memory. Using a paradigm in which participants interpreted direction and distance information to follow a mental path within a checkerboard grid, the present study shows that spatial framework results are obtained when reasoning occurs from a perspective that is misaligned with respect to the physical reference frame of the participant. The theoretical implications of these results are discussed.
\end{abstract}

Although spatial representations are typically constructed through direct interaction with the world, people can nevertheless reason about spaces learned about solely through language (e.g., when reading about a foreign city in a book). A growing literature in the field of spatial cognition has focused on how memories for spaces acquired through language are organized.

A series of influential studies by Tversky and her colleagues (e.g., Bryant, Tversky, \& Franklin, 1992; Franklin \& Tversky, 1990; Franklin, Tversky, \& Coon, 1992; see also Avraamides, 2003) have investigated how people organize in memory the space that surrounds their body. In the typical paradigm, participants first read narratives that describe themselves as central characters in settings where objects are located around them in canonical directions (i.e., above them, below them, and to their front, back, left, and right). After reading a sentence that reorients them in the imagined scene, the participants are probed with a directional term (e.g., left) and asked to respond with the name of the object that occupies the probed direction. Results from several studies have shown that response times vary systematically depending on the egocentric locations of objects. Specifically, in the case of an upright central character, objects located on the head-feet axis are responded to faster than those on the front-back axis, which are in turn accessed faster than objects on the left-right axis. Furthermore, an asymmetry is observed for the front-back axis: Objects located in front of the central character are retrieved faster than those in back.

Franklin and Tversky (1990) have proposed the spatial framework model to account for the observed pattern of latencies. They suggested that people use the extensions

We are grateful to Rich Carlson and three anonymous reviewers for providing valuable comments and suggestions on an earlier draft of this article. Correspondence relating to this article may be sent to M. N. Avraamides, Department of Psychology, University of Cyprus, P.O. Box 20537, Nicosia 1678, Cyprus (e-mail: mariosav@ucy.ac.cy). of their body axes as the reference frame for encoding object locations in memory. According to this model, the accessibility of each egocentric direction is determined by the perceptual and functional characteristics of the body and the world that specify how salient each direction is (see also Clark, 1973).

In a series of four experiments, Mou, Zhang, and McNamara (2004) examined the reference systems involved in spatial memories derived from language. In their Experiment 1, participants read narratives describing four objects located around them in the canonical orientations of the horizontal plane. After physically turning to adopt a new orientation, the participants were asked to locate target objects by responding to directional terms (e.g., front, left, etc.). Before any responses were made in each orientation, however, participants' focus of attention was manipulated with the presentation of a sentence that described one object from the array (see also Hintzman, O'Dell, \& Arndt, 1981, for a similar manipulation). The results revealed two independent effects. First, the spatial framework pattern of results (i.e., front $<$ back $<$ left $=$ right) was obtained. Second, a focus-of-attention effect was found: Participants were fastest when locating the object that was in the focus of their attention, intermediately fast with the object opposite that focus, and slowest with objects to the left or right of the attention focus. Mou et al.'s Experiment 3 was identical to their Experiment 1, except that participants located objects by responding to iconic arrows rather than directional terms. In contrast to Experiment 1, however, Experiment 3 revealed no spatial framework results (see also de Vega, Rodrigo, \& Zimmer, 1996, for similar findings), leading the authors to conclude that spatial framework results are not produced because of how locations are represented in memory, but instead derive from processes related to the interpretation of directional terms.

Mou et al. (2004) then proposed a two-step model for searching imagined spaces. In the first step, people interpret spatial cues to determine where to search. If the cues 
are directional terms (as in the majority of spatial framework studies), the perceptual and functional characteristics of our body and the world, as proposed by Franklin and Tversky (1990), determine the ease with which the step is completed. In the second step, people proceed to identify the object at the cued location.

Results that can be accounted for by Mou et al.'s (2004) model are provided by an earlier study conducted by Avraamides and Carlson (2003). Participants in that study were presented with a $12 \times 12$ checkerboard grid and were asked to mentally trace a path in it by continuously interpreting directions and distances (e.g., left three squares). In one condition of the experiment, termed the constantorientation condition, participants interpreted each directional term using a reference frame that was centered on their physical body (e.g., left always meant "move in the direction of your own physical left"). Because the physical orientation of participants remained unchanged, the reference frame used to interpret the directional terms was kept constant throughout the experiment. In another condition, termed the updated-orientation condition, the directional terms were interpreted on the basis of an imagined egocentric reference frame (i.e., left meant "move to the left of your imagined facing direction"). When moving in a given direction, the participants were instructed to imagine facing toward that direction at the end of the step and to interpret the next directional term based on that imagined facing direction. Because facing direction could vary on each step of the path, the meaning of directional terms needed to be frequently assessed.

Results for the updated-orientation condition followed the spatial framework pattern: Participants were fastest moving to the front, intermediately fast moving to the back, and slowest moving to the left or right. The results from the constant-orientation condition, however, deviated from the spatial framework pattern. In that condition, moving to the front was the fastest, but moving to the back was now slower than moving to the left or right.

Obtaining the spatial framework pattern in that experiment was a noteworthy result, since it extended the applicability of spatial frameworks to novel situations entailing no responses to target objects held in memory. However, the absence of the expected pattern in the constant-orientation condition indicates that caution should be used in applying the model.

Avraamides and Carlson (2003) argued that the occasional reorienting of the facing direction might be an important prerequisite for spatial framework results, since it forces participants to continuously evaluate the mappings between directional terms and the space (previous studies using the spatial framework paradigm typically involved reorientations of an imagined egocentric reference frame). This is in line with the postulates of Mou et al.'s (2004) model. When directional terms had to be interpreted in every step of the task, with the functional and perceptual invariants influencing this process, spatial frameworks emerged. In contrast, the constant-orientation condition did not require continuous interpretation of directional terms. Therefore, participants could interpret directional terms once and simply maintain the same mappings of terms to a space throughout the experiment. If this analysis is indeed correct, one would expect the influence of perceptual and functional invariants on latencies (if any) to be minimal in the constant-orientation condition. One plausible hypothesis, then, is that the spatial framework pattern is limited to cases in which the assignment of directional labels to space is frequently changed.

An alternative account is possible, however. A plethora of studies in spatial cognition have shown that people reason more efficiently about space when they do so from their physical viewpoint rather than from a novel one adopted by using imagination (see, e.g., Avraamides, Klatzky, Loomis, \& Golledge, 2004; Presson \& Montello, 1994; Rieser, 1989). In the former case, people execute tasks such as locating targets from memory by relying on a primary reference frame that is centered on their body (Presson \& Montello, 1994). In the latter case, people need to establish a secondary reference frame, project it onto the imagined location, and orient it according to the instructed imagined facing direction. As several studies have shown, difficulties arise when the primary and secondary reference frames are misaligned (see May, 2004, for an account based on interference). Furthermore, the difficulties are even greater when the task involves interpreting the directional terms left and right (Avraamides, 2003). Because Avraamides and Carlson's (2003) updatedorientation condition (unlike their constant-orientation condition) presumably entailed constructing a secondary imagined reference frame that was misaligned with the physical body more often than not, one would expect that (1) performance would be overall inferior in the updatedorientation condition, and (2) moving to the left or right would be particularly slow in that condition, thus contributing to the emergence of spatial frameworks. Both of these findings were reported by Avraamides and Carlson.

The goal of the present experiment was to contrast the two accounts sketched above. Specifically, we aimed to examine whether a frequently updated reference frame is indeed a prerequisite for spatial frameworks, or whether a misaligned imagined reference frame alone would suffice. We have modified the task from Avraamides and Carlson (2003) to include conditions in which participants interpret directional terms using reference frames that are constant but misaligned with respect to the physical reference frame. For example, one group of participants were asked to imagine themselves starting out facing to the right $\left(90^{\circ}\right.$ clockwise misalignment with respect to their physical facing direction) and to keep facing to the right when completing each movement. When presented, for example, with the direction front, they had to imagine moving not to their own physical front, but to the front of their imagined facing direction. If occasional updating of the facing orientation is indeed required for spatial frameworks, the spatial framework pattern of results should not be obtained in these constant-orientation conditions. If, however, spatial frameworks require only the construction 
of a secondary, misaligned egocentric reference frame, the spatial framework pattern of results should then be expected.

\section{METHOD}

\section{Participants}

Fifty-one students from an introductory psychology course participated in the experiment in exchange for course credit. Fifteen of the participants were randomly assigned to the aligned orientation condition, and 12 to each of the three misaligned orientation conditions.

\section{Materials}

The stimuli were presented on 19-in. computer monitors that were fitted in custom-made stands to face upward, allowing an oblique viewing angle ranging from $45^{\circ}$ to $60^{\circ}$, depending on a participant's height. The participants were seated in front of the monitors at a comfortable distance. The task was programmed and presented using the MEL 2 software package.

As seen in Figure 1, the display included the presentation of a $12 \times 12$ grid that alternated between light and dark $1.3-\mathrm{cm}$ squares. Information about direction and distance appeared in a green circle of $1.3-\mathrm{cm}$ diameter that was presented in the center of the display.
The participants proceeded through displays using a mouse that was conveniently placed on a table to the right of the monitor.

\section{Design}

The experiment followed a 4 (orientation misalignment: $0^{\circ}, 90^{\circ}$ clockwise, $90^{\circ}$ counterclockwise, or $\left.180^{\circ}\right) \times 4$ (direction: front, back, left, or right) $\times 2$ (order of information: direction-first or distance-first) mixed factorial design. Orientation misalignment was manipulated between subjects, and direction and the order of information were manipulated within subjects.

\section{Procedure}

The participants completed 2 practice trials, followed by five blocks of 12 experimental trials. Trials required that the participants interpret presented directions (front, back, left, or right) and distances $(2,3,4$, or 5 squares $)$ in order to mentally move around the grid from a given starting point. Each trial included eight steps (i.e., eight direction-distance pairs), with each direction and distance sampled randomly twice within the trial. The participants were instructed to respond as fast as they could without sacrificing accuracy for speed.

Trials began with the presentation of the grid. When a participant first clicked with the mouse, the starting location appeared as a red square. The starting location was selected randomly on each trial,

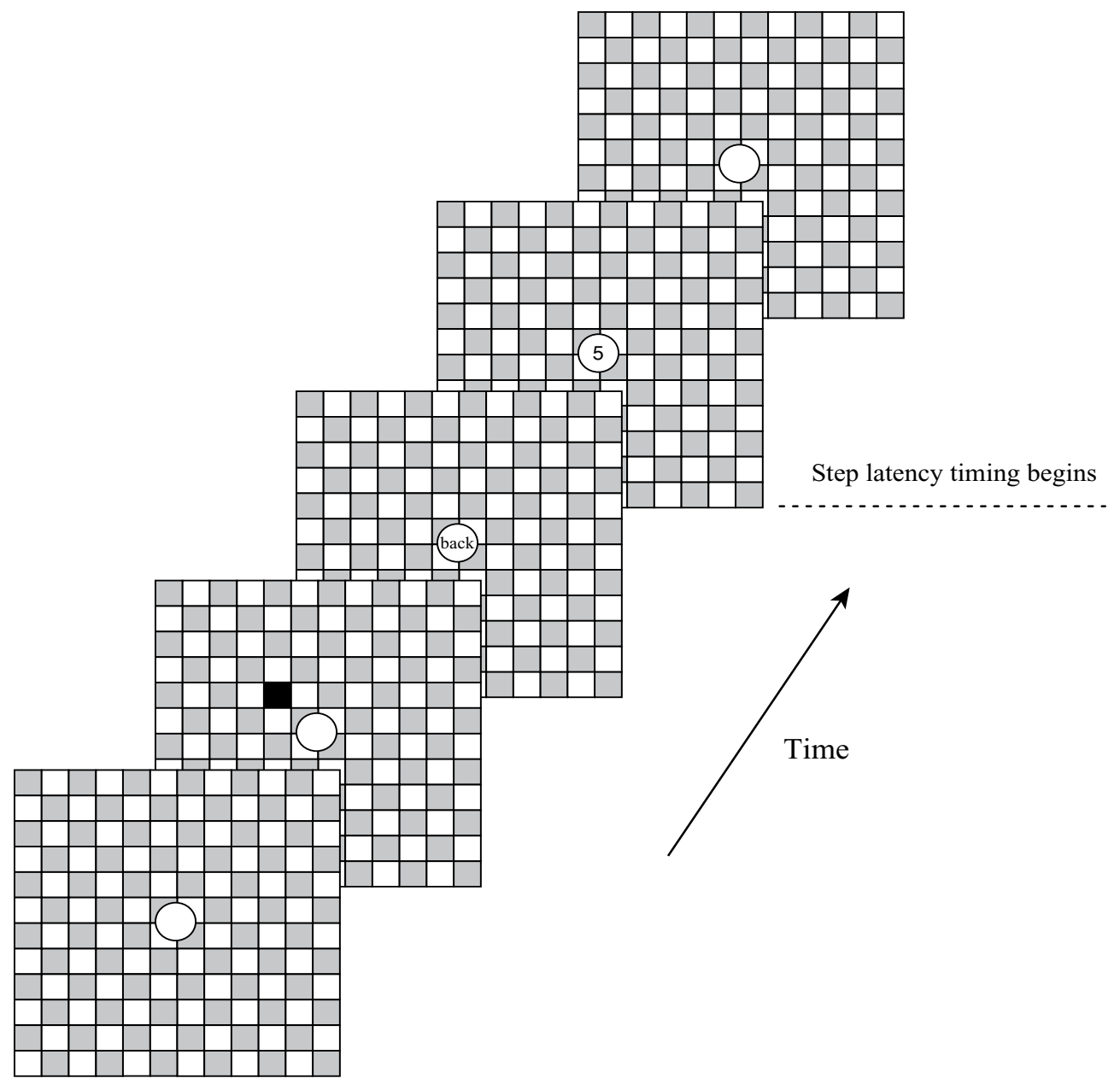

Figure 1. Schematic depiction of the time course of the first step of a trial. 
with the constraint that it was never one of the outermost squares of the grid. On each subsequent mouse click, information about direction and distance appeared in the green circle in the center of the display. The program ensured that no direction-distance information would lead the path outside the grid. The order in which direction and distance were presented varied from trial to trial but remained constant within each trial. In half of the trials, direction preceded distance, and in the other half, direction was presented after distance. At the end of the eighth step, a check mark appeared and the participant was asked to move it with the mouse and click on the ending location of the path, and thus indicate their response.

The mouse cursor remained invisible throughout the experiment, and the participants were not allowed to touch the display to mark either the starting or any intermediate locations on the grid.

The participants in all orientation conditions were instructed to visualize the grid as a tiled floor that they could stand on and move around. Conditions differed with respect to the imagined orientation the participants were asked to maintain while navigating the grid. In the aligned orientation $\left(0^{\circ}\right.$ misalignment $)$ condition, they were asked to imagine themselves facing the same direction they were actually facing as they were physically seated in front of the display. Therefore, in this condition the spatial labels front, back, left, and right corresponded in both the imagined egocentric reference frame and the physical reference frame of the participant. When, for example, the direction front was presented, the participants had to imagine moving to the front as indicated by their own physical position. In the three misaligned orientation conditions $\left(90^{\circ}\right.$ clockwise, $90^{\circ}$ counterclockwise, and $180^{\circ}$ misalignment), the participants were asked to imagine themselves on the grid facing a direction other than the one their physical body faced. In the $90^{\circ}$ clockwise condition, they imagined facing toward their own right. Therefore, when the direction front was presented, they had to imagine moving to the right. In the $90^{\circ}$ counterclockwise and $180^{\circ}$ misalignment conditions, they imagined facing toward the left and back, respectively. Figure 2 shows the orientation of the imagined facing direction in each condition.

\section{RESULTS}

The first block of trials was considered practice and was excluded from all analyses. All trials in which the participant indicated an endpoint that was not correct were considered errors. For 1 participant in the $180^{\circ}$ misalignment condition, all data were discarded because of very low accuracy $(<10 \%)$. Overall, participants responded correctly to $71 \%$ of the trials. A repeated measures ANOVA on errors using orientation misalignment and order of information as factors revealed no significant effects or interactions. Therefore, step latency data became the primary focus.

Latencies were recorded on each of the eight steps of each trial. Timing began when all the information in the step became available - that is, when the distance was presented in the direction-first trials and when the direction was presented in the distance-first trials. Timing ended when a participant clicked with the mouse to request the direction-distance pair for the next step or the check mark in the eighth step. Initial analyses revealed that latencies did not differ between left and right, so data from those two directions were combined to form a left-right direction level.

A repeated measures ANOVA revealed that step latencies were shortest in the $0^{\circ}$ misalignment condition $(2,261 \mathrm{msec})$, intermediate in the $90^{\circ}$ conditions $(2,726$
A
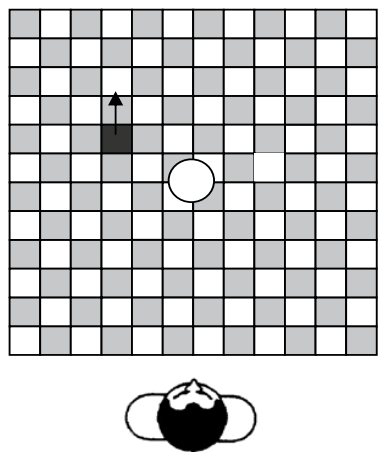

C
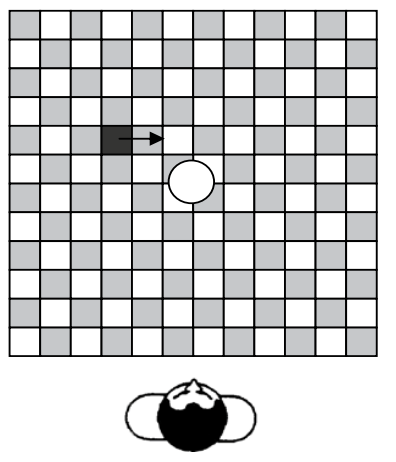

B
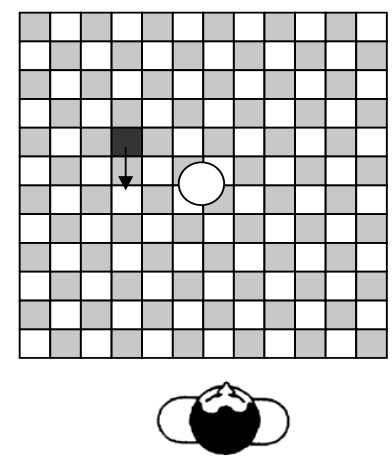

D
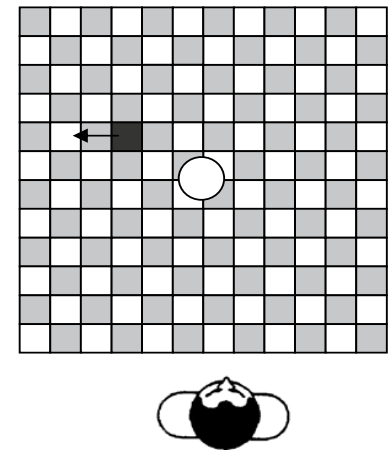

Figure 2. Orientation of imagined egocentric reference frames, indicated in each panel by the arrow (not visible to participants) on the starting square. Panels A-D show, respectively, orientation to the imagined facing direction in the $0^{\circ}, 180^{\circ}, 90^{\circ}$ clockwise, and $90^{\circ}$ counterclockwise misalignment conditions.

and 2,704 msec, respectively, for clockwise and counterclockwise), and longest in the $180^{\circ}$ condition $(3,087 \mathrm{msec})$ $\left[F(3,46)=2.84, M S_{\mathrm{e}}=3,021,743, p<.05\right]$. Pairwise $t$ tests confirmed that all differences were significant $(p \mathrm{~s}<.05)$.

A main effect of direction was also obtained $[F(2,92)=$ $\left.59.25, M S_{\mathrm{e}}=143,432, p<.001\right]$. However, direction interacted significantly with orientation misalignment $\left[F(6,92)=4.96, M S_{\mathrm{e}}=143,432, p<.001\right]$. As seen in Figure 3, the interaction resulted from the fact that the pattern of step latencies was different in the $0^{\circ}$ misalignment condition than in the remaining conditions. In the $0^{\circ}$ condition, latencies were shortest for moving to the front, intermediate for moving to the left or right, and longest for moving to the back. Pairwise $t$ tests revealed that all differences were significant ( $p$ s $<.01$ ). The pattern was substantially different, however, in the remaining conditions. In those conditions, moving to the front was again the fastest, but moving to the back was faster than moving either left or right (all $p \mathrm{~s}<.05$ ).

Furthermore, step latencies were shorter for directionfirst trials $(2,645 \mathrm{msec})$ than for distance-first trials $(2,743 \mathrm{msec})\left[F(1,46)=8.2, M S_{\mathrm{e}}=89,231, p<.01\right]$. The order of information did not interact with either orientation misalignment or direction. 


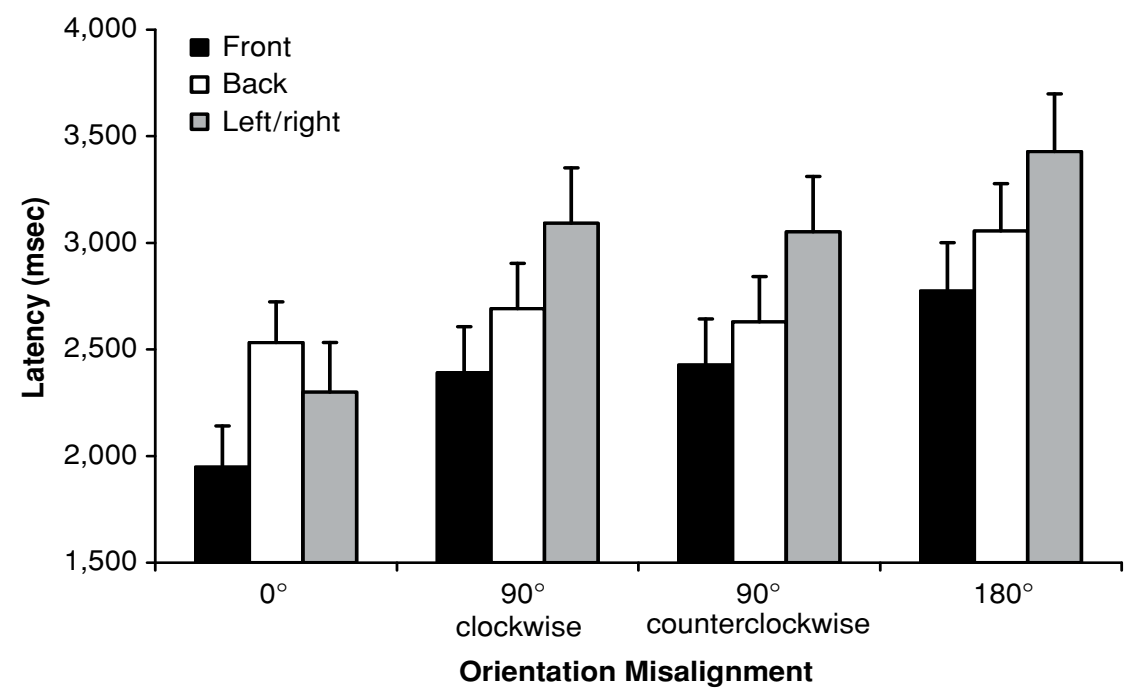

Figure 3. Step latency as a function of orientation misalignment and movement direction. Error bars represent betweeen-subjects standard errors.

\section{DISCUSSION}

The present experiment contrasted the predictions of two hypotheses concerning the conditions that are necessary for spatial framework results to emerge. One hypothesis was that spatial framework results occur only when an imagined facing orientation is frequently updated, because in this case the egocentric directional terms have to be continuously interpreted. An alternative hypothesis states that the spatial framework model applies in cases in which a misaligned imagined egocentric reference frame is used, because in this case interpreting directional terms, and especially the terms left and right, is difficult. Our results clearly support the latter hypothesis. Spatial framework results were obtained in three conditions in which an imagined reference frame, misaligned with the physical reference frame but constant throughout the experiment, was needed. In all three conditions, mentally moving to the front was faster than moving to the back, which in turn was faster than moving to the left or right. On the other hand, in the one condition in which participants interpreted directional terms based on their physical egocentric reference frames, a different result pattern was obtained. Replicating the previous results of Avraamides and Carlson (2003) for this condition, moving to the front was faster than moving to the left or right, which in turn was faster than moving to the back.

The misaligned reference frame conditions in the present experiment provide an intermediate situation between the constant-orientation and updated-orientation conditions of Avraamides and Carlson (2003). Participants needed to construct a secondary, imagined reference frame and orient it in a direction that was not parallel to their primary physical reference frame; this orientation, however, remained constant throughout the experiment. One could, therefore, wonder why the spatial framework pattern arose if (because of the constant nature of the ref- erence frame) the interpretation of directional terms took place only once. In other words, participants could have constructed an imagined reference frame, used it to map directional cues to the space, and maintained this reference frame along with the mappings in memory for the rest of the experiment. Results from the literature suggest, however, that people do not readily maintain abstract imagined reference frames in memory, but instead prefer to construct them ad hoc when needed (see, e.g., Hintzman et al., 1981; Shepard \& Hurwitz, 1984). For example, in an experiment in which participants judged whether turns were to the right or left in simplified map displays, Shepard and Hurwitz found that participants did not spontaneously use advance information about the orientation of the path leading to the turn to construct and maintain an anticipatory imagined egocentric reference frame. On the basis of this previous evidence, then, it is likely that participants in the present experiment did not maintain the misaligned reference frame throughout the experiment but instead reconstructed it each time a directional cue was presented.

Results from the aligned condition in the present experiment replicated those of the constant-orientation condition of Avraamides and Carslon (2003); this is of course not surprising, since the two conditions were identical. The pattern obtained deviated from that for spatial frameworks, in that front was the fastest direction but back was slower than left or right. Overall, latencies were shorter than those in the three misalignment conditions. In fact, latencies became longer as orientation misalignment increased. This finding is in line with previous studies examining memory for spatial layouts, which have shown that spatial performance typically suffers when reasoning takes place from imagined perspectives that are misaligned with one's physical perspective (e.g., Avraamides \& Kelly, 2005; May, 2004; Rieser, 1989). In an extension of the previous studies, our results show that problems 
with perspective misalignment exist even when reasoning takes place in a perceptual scene that contains no objects.

However, what still needs to be accounted for is the specific pattern of latencies in the constant-orientation condition. We believe that participants interpreted directional terms using their physical reference frame and maintained the mappings of directional terms to the space throughout the course of the experiment. If this was the case, only modest (or no) costs due to directional cue interpretation (i.e., the first step in Mou et al.'s [2004] model) should be expected. In this situation, one could regard left and right as being particularly fast, as opposed to back, which was particularly slow. If directional cue interpretation is not a costly process in the case of the aligned facing direction, then the latency differences for the various egocentric directions that were observed should have stemmed from processes involved in accessing the imagined space (the second step in Mou et al.'s model). It is possible, for example, that participants focused their attention first in the frontal direction and then shifted it in a manner analogous to physically turning toward the direction indicated by the directional cue. This, or a similar strategy, could give rise to the specific pattern of latencies evidenced in this condition. More research will definitely be required before conclusions can be reached.

In summary, the results from the present experiment suggest that frequent reorientation of an egocentric perspective is not a prerequisite for spatial frameworks, as was suggested by Avraamides and Carlson (2003). Instead, reasoning from an imagined perspective that is misaligned with the physical reference frame suffices for spatial framework results to emerge. We have argued that this is the case because when reasoning from misaligned perspectives, people exhibit difficulties in interpreting directional terms, and especially those involving the left-right dimension. The finding of the spatial framework pattern in three of our conditions suggests that (1) spatial frameworks are not limited to situations in which the locations of objects are maintained in memory and then responded to, and (2) spatial frameworks arise not from the processes involved in representing locations in memory, but are instead a product of the processes involved in interpreting direction terms.

\section{REFERENCES}

AvraAmides, M. N. (2003). Spatial updating of environments described in texts. Cognitive Psychology, 47, 402-431.

AvraAmides, M. N., \& CARlson, R. A. (2003). Egocentric organization of spatial activities in imagined navigation. Memory \& Cognition, 31, 252-261.

Avraamides, M. N., \& Kelly, J. W. (2005). Imagined perspectivechanging within and across novel environments. In C. Freksa, M. Knauff, B. Krieg-Brückner, B. Nebel, \& T. Barkowsky (Eds.), Spatial cognition IV: Reasoning, action, interaction (Lecture Notes in Computer Science, Vol. 3343, pp. 245-258). Berlin: Springer.

Avraamides, M. N., Klatzky, R. L., Loomis, J. M., \& Golledge, R. G. (2004). Use of cognitive versus perceptual heading during imagined locomotion depends on the response mode. Psychological Science, 15, 403-408.

Bryant, D. J., Tversky, B., \& Franklin, N. (1992). Internal and external spatial frameworks for representing described scenes. Journal of Memory \& Language, 31, 74-98.

Clark, H. H. (1973). Space, time, semantics, and the child. In T. E. Moore (Ed.), Cognitive development and the acquisition of language (pp. 27-63). New York: Academic Press.

de Vega, M., Rodrigo, M. J., \& Zimmer, H. (1996). Pointing and labeling directions in egocentric frameworks. Journal of Memory \& Language, 35, 821-839.

Franklin, N., \& TVersky, B. (1990). Searching imagined environments. Journal of Experimental Psychology: General, 119, 63-76.

Franklin, N., TVersky, B., \& CoON, V. (1992). Switching points of view in spatial mental models. Memory \& Cognition, 20, 507-518.

Hintzman, D., O’Dell, C., \& ARndt, D. (1981). Orientation in cognitive maps. Cognitive Psychology, 13, 149-206.

MAY, M. (2004). Imaginal perspective switches in remembered environments: Transformation versus interference accounts. Cognitive Psychology, 48, 163-206.

Mou, W., Zhang, K., \& McNamara, T. P. (2004). Frames of reference in spatial memories acquired from language. Journal of Experimental Psychology: Learning, Memory, \& Cognition, 30, 171-180.

Presson, C. C., \& Montello, D. R. (1994). Updating after rotational and translational body movements: Coordinate structure of perspective space. Perception, 23, 1447-1455.

RIESER, J. J. (1989). Access to knowledge of spatial structure at novel points of observation. Journal of Experimental Psychology: Learning, Memory, \& Cognition, 15, 1157-1165.

SHEPARD, R. N., \& HuRWITZ, S. (1984). Upward direction, mental rotation, and discrimination of left and right turns in maps. Cognition, 18, 161-193.

(Manuscript received June 30, 2005; revision accepted for publication October 28, 2005.) 